\title{
Blood Lactate Dehydrogenase Increased, CTCAE
}

National Cancer Institute

\section{Source}

National Cancer Institute. Blood Lactate Dehydrogenase Increased, CT CAE. NCI

Thesaurus. Code C143218.

A finding based on laboratory test results that indicate increased levels of lactate dehydrogenase in the blood specimen. 